\title{
BMJ Open Does Gleason score of positive surgical margin after radical prostatectomy affect biochemical recurrence and oncological outcomes? Protocol for systematic review
}

\author{
Athul John (D) ,1,2 Michael O'Callaghan, ${ }^{1,3,4}$ Rick Catterwell, ${ }^{1,2}$ Luke A Selth ${ }^{1,4}$
}

To cite: John A, O'Callaghan M, Catterwell R, et al. Does Gleason score of positive surgical margin after radical prostatectomy affect biochemical recurrence and oncological outcomes? Protocol for systematic review. BMJ Open 2020;10:e034612. doi:10.1136/ bmjopen-2019-034612

- Prepublication history for this paper is available online. To view these files, please visit the journal online (http://dx.doi. org/10.1136/bmjopen-2019034612).

Received 04 0ctober 2019 Revised 08 February 2020 Accepted 04 March 2020

Check for updates

(C) Author(s) (or their employer(s)) 2020. Re-use permitted under CC BY-NC. No commercial re-use. See rights and permissions. Published by BMJ.

For numbered affiliations see end of article.

Correspondence to

Dr Athul John;

atuljohn@gmail.com

\section{ABSTRACT}

Introduction Positive surgical margins (PSM) in cancer patients are commonly associated with worse prognosis and a higher risk of secondary treatment. However, the relevance of this parameter in prostate cancer patients undergoing radical prostatectomy $(\mathrm{RP})$ remains controversial, given the inconsistencies in its ability to predict biochemical recurrence (BCR) and oncological outcomes. Hence, further assessment of the utility of surgical margins for prostate cancer prognosis is required to predict these outcomes more accurately. Over the last decade, studies have used the Gleason score (GS) of positive margins to predict outcomes. Herein, the authors aim to conduct a systematic review investigating the role of GS of PSM after radical prostatectomy in predicting BCR and oncological outcomes.

Methods and analysis We will perform a search using MEDLINE, EMBASE, SCOPUS and COCHRANE databases.

The review will be reported according to the Preferred Reporting Items for Systematic Reviews and Meta-Analyses guidelines. We will screen titles and abstracts to select articles appropriate for full-text review. Studies discussing GS of PSM after RP will be included. Given the change in reporting of GS, only articles from 2005 to 2019 will be included. The quality of the studies chosen will be assessed using the Newcastle Ottawa tool for non-randomised and Cochrane risk of bias for randomised control studies. We will adopt the grading of recommendations, assessment, development and evaluation framework to comment on quality of cumulative evidence. The primary outcome measure will be time to BCR. Secondary outcome measures include secondary treatment, disease-specific survival, disease progression-free and overall mortality at follow-up period. We aim to perform a meta-analysis if the level of heterogeneity is acceptable $\left(1^{2}<50 \%\right)$.

Ethics and dissemination The review does not require ethics approval as it is a review of published literature. The findings of the review will be submitted for peer-reviewed publications and presented at scientific meetings. PROSPERO registration number CRD42019131800.

\section{INTRODUCTION}

Positive surgical margins in cancer patients are commonly associated with worse prognosis
Strengths and limitations of this study

- Positive surgical margin after radical prostatectomy remains controversial in its ability to predict longterm outcomes after surgery.

- To our knowledge, this is the first systematic review and meta-analysis investigating outcomes of patients after radical prostatectomy based on Gleason score of positive surgical margin site.

- A major limitation of the study is lack of randomised controlled trials, and the majority of expected studies are likely to be retrospective cohort studies.

- Quality assessment of included studies will also be reported.

- The Preferred Reporting Items for Systematic Reviews and Meta-Analyses protocol checklist will be followed when reporting the findings.

and a higher risk of secondary treatment. However, its role in patients undergoing radical prostatectomy remains controversial since only $30 \%-35 \%$ and $19 \%-48 \%$ of men with positive surgical margins develop metastatic disease or biochemical recurrence after radical prostatectomy, respectively. ${ }^{12}$ Positive surgical margins have been reported in $11 \%-40 \%$ of men undergoing radical prostatectomy. Given the apparent inaccuracy of positive surgical margins as a means to predict prostate cancer progression, further evaluation of this parameter is required to improve its predictive value.

Recently, there have been multiple studies investigating Gleason score of positive surgical margins and its impact on biochemical recurrence. ${ }^{1-6}$ As a result, some studies recommend mandatory reporting of Gleason score of positive surgical margin. This is in contrast to the current International Society of Urological Pathology recommendation, which leaves the decision up to the discretion of the pathologist. 
However, very few studies report oncological outcomes and the relationship between biochemical recurrence and long-term survival rates are still poorly defined. Hence, the authors aim to conduct a systematic review investigating the role of Gleason score of positive surgical margins after radical prostatectomy in predicting biochemical recurrence and oncological outcomes (eg, cancer-specific survival and all-cause survival). To the authors' knowledge, no systematic reviews have explored this topic previously.

\section{REVIEW QUESTION}

In men who have positive surgical margins after radical prostatectomy, does a low Gleason score at the margin compared with a high Gleason score affect biochemical recurrence and long-term oncological outcomes?

\section{OBJECTIVE}

To conduct a systematic review investigating the role of Gleason score at positive surgical margin site in men who have undergone radical prostatectomy for prostate cancer, in predicting biochemical recurrence and longterm oncological outcomes.

\section{METHODS}

\section{Eligibility criteria}

The search strategy aims to find published studies exploring the role of Gleason score of positive surgical margins after radical prostatectomy in predicting biochemical recurrence and oncological outcomes. The review will consider all published studies, including metaanalysis and randomised controlled trials; however, we will also consider observational cohort studies and casecontrolled studies if level 1 evidence is not available.

Language will be restricted to English. Studies with men who underwent radical prostatectomy without reporting of Gleason score at positive surgical margin site will be excluded. Grey literature, including conference abstracts and editorials, will be excluded.

\section{Patient and public involvement}

No patient involved.

\section{Information sources}

The review will involve searching the MEDLINE, SCOPUS, EMBASE and COCHRANE databases. Given the change in Gleason reporting in 2005, the review will only include studies published between 1 January 2005 and 31 September 2019. ${ }^{7}$ A further comprehensive literature search will also involve examining reference lists of included studies identified from the search. Authors will be contacted if the published study does not contain sufficient details to extract data.

\section{Search strategy}

The search strategy will be created with the assistance of health sciences librarians with previous expertise in conducting systematic searches. The search strategies will be modified to accommodate the requirements of different databases used for the search.

A draft of MEDLINE (OVID interface) search strategy is shown in table 1 .

\section{STUDY RECORDS}

\section{Data management}

A preformulated data extraction template will be used to keep track of information obtained from each study. Software including Covidence (Covidence systematic review software, Veritas Health Innovation, Melbourne, Australia) and EndNote X8.2 will be used to track studies included and excluded from the review. Covidence will also be used to assist with tracking the quality of assessment and extracted information. This data will be tabulated using Microsoft Excel (Redmond, Washington, USA).

Table 1 Search terms for MEDLINE

\begin{tabular}{|c|c|c|c|}
\hline Population & Intervention & Comparators & Outcomes \\
\hline Men with prostate cancer & Radical prostatectomy & $\begin{array}{l}\text { Gleason score at positive } \\
\text { surgical margin site }\end{array}$ & $\begin{array}{l}\text { Biochemical recurrence and oncological } \\
\text { outcomes }\end{array}$ \\
\hline
\end{tabular}




\section{Data collection and selection process}

A comprehensive search strategy aims to find published studies in various electronic databases, including the MEDLINE, SCOPUS, EMBASE and COCHRANE databases. Studies will be screened by two authors by titles and abstracts to determine if it is appropriate. Once screened, the full-text article will be retrieved. If inclusion criteria is fulfilled, it will be selected for the review. Hand searching of reference lists of the selected studies will also be conducted and be considered for inclusion based on inclusion criteria based on the same criteria. Any disagreements between authors will be discussed with a third reviewer. Once included, the authors aim to extract, tabulate and summarise details of the eligible studies.

\section{DATA ITEMS}

Study characteristics to be extracted by the review include title, study design and type, financial supports, first authors, year study published, inclusion criteria, follow-up period and the period of enrolment for the study. Population characteristics include sample size, average age of men, year of surgery, age at diagnosis, body mass index and median postoperative follow-up. Intervention characteristics to be extracted type of procedure (robot-assisted, laparoscopic or open), year of surgery and additional interventions. Comparator characteristics include primary Gleason score at positive surgical margin, overall Gleason score at margin, Gleason grade group of specimen, Gleason grade group at margin, surgical margin length of invasion, Gleason score on biopsy, extent of margin, lobe of prostate cancer, location of margin, Extraprostatic extension, perineural invasion, lymphadenopathy, pT stage and Prostate Specific Antigen (PSA) at diagnosis. Outcome characteristics include biochemical recurrence, secondary treatment rate, survival postsurgery, number of individuals with metastasis and systemic progression at median/mean follow-up, systemic progression-free survival.

\section{OUTCOMES AND PRIORITISATION}

The review will aim to extract and report following outcome measures in the following patterns.

\section{Primary outcomes measure}

Time to biochemical recurrence after radical prostatectomy. Biochemical recurrence is defined by two consecutive PSA values of $>0.2 \mathrm{ng} / \mathrm{mL}$ and rising. ${ }^{8}$ This is one of the main indicators used in clinical practise to commence secondary treatment and to commence assessment of metastatic spread.

\section{Secondary outcome measures}

Prostate cancer-related mortality. This is defined as death as a result of prostate cancer in the cohort. Given the chronicity prostate cancer, studies should look specifically at prostate cancer-related mortality rate to avoid other confounders which may also cause death in individuals involved in the cohort study. This may also be reported as an HR. This will be more beneficial than the overall survival rate.

\section{Secondary treatment/intervention rate}

The number of individuals who required additional treatment for prostate cancer such as Androgen deprivation therapy or external beam radiotherapy after the radical prostatectomy. This outcome is dependent on biochemical recurrence. Repeated treatments have been associated with worse comorbidities; hence, this outcome is important to establish so that patients can be counselled appropriately.

\section{Cancer-free survival at follow-up}

Number of participants that are alive and have no biochemical recurrence at follow-up period of the study.

Metastasis free survival or systemic progression-free at follow-up Number of participants that are alive have no evidence of prostate cancer metastasis or systemic progression at follow-up period of the study.

\section{Outcome follow-up periods}

All mean and median follow-up period will be noted. Based on initial searches, studies are likely to have significant variability in the short-term and long-term follow-up periods. Studies with identical follow-up periods will be considered for a meta-analysis. If time-specific estimates are not provided, we hope to report HRs. The authors agree that a median follow-up of less than 12 months is inadequate in regards to detecting biochemical recurrence postradical prostatectomy.

\section{RISK OF BIAS IN INDIVIDUAL STUDIES}

The quality of the studies chosen would be assessed using the Newcastle Ottawa tool which is used for assessing the quality of non-randomised studies included in a systematic review by assessing domains such as selection of study groups, comparability of the groups and based on exposure or outcome of interest. Stars are awarded for each domain which allows the study to be graded into poor, fair or good quality. ${ }^{9}$ For randomised control trials, Cochrane risk of bias tools will be used to assess the bias. A funnel plot will be used to represent an assessment of publication bias.

\section{DATA SYNTHESIS}

The authors aim to summarise the role of Gleason score of positive surgical margins after radical prostatectomy in predicting biochemical recurrence and long-term oncological outcomes. Cox proportional-HRs of both multivariate and univariate analysis data on primary and secondary outcomes would be extracted. This data will be presented as forest plots. The heterogeneity of the selected studies would be calculated using the $\mathrm{I}^{2}$ score. Meta-analysis will 
use a random-effects model as the studies extracted are likely to have some differences in the way Gleason score is grouped. If there is considerable heterogeneity, sources of heterogeneity will be explored, and further subgroup analysis would be conducted using various Gleason scores at positive surgical margin. ${ }^{10}$ The outcome measures would be summarised in a tabular format. We will use the Preferred Reporting Items for Systematic Reviews and Meta-Analyses checklist when writing our report. ${ }^{11}$

\section{Confidence in cumulative evidence}

The authors believe oncological outcomes such as cancerfree survival, disease progression and survival should be followed up for a minimum of 5 years postradical prostatectomy. The authors would also evaluate and critically appraise studies adjusted for confounders such as age of diagnosis, prediagnosis PSA and biopsy Gleason grade and any additional therapy before surgery. Overall, the authors aim to adopt the grading of recommendations, assessment, development and evaluation framework to assess each outcome measure to comment on quality of cumulative evidence. $^{12}$

\section{Dissemination plans}

The authors aim to publish the review in a peer-reviewed scientific journal and present thefindings at relevant national and international scientific meetings.

\section{Author affiliations}

${ }^{1}$ Adelaide Medical School, The University of Adelaide Faculty of Health and Medical Sciences, Adelaide, South Australia, Australia

${ }^{2}$ Urology, Central Adelaide Local Healthcare Network, Adelaide, South Australia, Australia

${ }^{3}$ Urology, South Australia Prostate Cancer Clinical Outcomes Collaborative, Adelaide, South Australia, Australia

${ }^{4}$ Flinders Centre for Innovation in Cancer, College of Medicine and Public Health, Flinders University, Adelaide, South Australia, Australia

Correction notice This article has been corrected since it was published. The affiliations have been updated.

Contributors AJ and MOC drafted the manuscript and created the study concept of the systematic review. RC and LAS provided supervision and guidance during the formulation of the study. All authors were also involved in reviewing and critically appraising the protocol in its current form. The authors acknowledge Vikki Langton for her assistance with the formulation of search strategy.

Funding The authors have not declared a specific grant for this research from any funding agency in the public, commercial or not-for-profit sectors.

Competing interests None declared.
Patient and public involvement Patients and/or the public were not involved in the design, or conduct, or reporting, or dissemination plans of this research.

Patient consent for publication Not required.

Provenance and peer review Not commissioned; externally peer reviewed.

Open access This is an open access article distributed in accordance with the Creative Commons Attribution Non Commercial (CC BY-NC 4.0) license, which permits others to distribute, remix, adapt, build upon this work non-commercially, and license their derivative works on different terms, provided the original work is properly cited, appropriate credit is given, any changes made indicated, and the use is non-commercial. See: http://creativecommons.org/licenses/by-nc/4.0/.

ORCID iD

Athul John http://orcid.org/0000-0001-9081-2288

\section{REFERENCES}

1 Savdie R, Horvath LG, Benito RP, et al. High Gleason grade carcinoma at a positive surgical margin predicts biochemical failure after radical prostatectomy and may guide adjuvant radiotherapy. BJU Int 2012;109:1794-800.

2 Viers BR, Sukov WR, Gettman MT, et al. Primary Gleason grade 4 at the positive margin is associated with metastasis and death among patients with Gleason 7 prostate cancer undergoing radical prostatectomy. Eur Urol 2014;66:1116-24.

3 Udo K, Cronin AM, Carlino LJ, et al. Prognostic impact of subclassification of radical prostatectomy positive margins by linear extent and Gleason grade. J Urol 2013;189:1302-7.

4 Kates M, Sopko NA, Han M, et al. Importance of reporting the Gleason score at the positive surgical margin site: analysis of 4,082 consecutive radical prostatectomy cases. J Urol 2016;195:337-42.

5 Albadine R, Hyndman ME, Chaux A, et al. Characteristics of positive surgical margins in robotic-assisted radical prostatectomy, open retropubic radical prostatectomy, and laparoscopic radical prostatectomy: a comparative histopathologic study from a single academic center. Hum Pathol 2012;43:254-60.

6 Brimo F, Partin AW, Epstein Jl. Tumor grade at margins of resection in radical prostatectomy specimens is an independent predictor of prognosis. Urology 2010;76:1206-9.

7 Epstein JI, Amin MB, Reuter VE, et al. Contemporary Gleason grading of prostatic carcinoma: an update with discussion on practical issues to implement the 2014 International Society of urological pathology (ISUP) consensus conference on Gleason grading of prostatic carcinoma. Am J Surg Pathol 2017;41:e1-7.

8 Cornford P, Bellmunt J, Bolla M, et al. EAU-ESTRO-SIOG guidelines on prostate cancer. Part II: treatment of relapsing, metastatic, and castration-resistant prostate cancer. Eur Urol 2017;71:630-42.

9 Wells G, Shea B, O'Connell D, et al. The Newcastle-Ottawa scale (NOS) for assessing the quality of Non-Randomized studies in metaanalysis. Ottawa Health Research Institute Web site 2000.

10 Fergusson D, Laupacis A, Salmi LR, et al. What should be included in meta-analyses? an exploration of methodological issues using the ISPOT meta-analyses. Int J Technol Assess Health Care 2000;16:1109-19.

11 Moher D, Shamseer L, Clarke M, et al. Preferred reporting items for systematic review and meta-analysis protocols (PRISMA-P) 2015 statement. Syst Rev 2015;4:1.

12 Balshem H, Helfand M, Schünemann $\mathrm{HJ}$, et al. Grade guidelines: 3. rating the quality of evidence. J Clin Epidemiol 2011;64:401-6. 